\title{
Arbor
}

\section{La democracia tecnológica}

\section{Miguel A. Quintanilla}

Arbor CLXXIII, 683-684 (Noviembre-Diciembre 2002), 637-651 pp.

Al reconocerse que nuestros productos escapan ya a nuestro control, también ha entrado en crisis el sueño de la izquierda, según el cual la Historia puede hacerse conscientemente»

Oskar Lafontaine

Con estas palabras concluía Oskar Lafontaine, hace más de una década, su brillante ensayo sobre la sociedad del futuro. Un futuro que ya es presente, por cierto. Una de las notas que más llaman la atención de aquel ensayo es el énfasis que el autor pone en la relación entre tecnología y política progresista, una herencia casi olvidada de la tradición ilustrada. Estas páginas tienen la pretensión de contribuir también a recuperar esa herencia, conectándola con otro de los temas recurrentes en el pensamiento progresista de finales del siglo XX: el de la extensión de la democracia.

Podemos considerar los posibles des̉arrollos de la democracia en torno a tres ejes. Uno de los ejes representa la extensión de la democracia, que puede ser tanto en sentido espacial como sectorial. Aquí hay un amplio margen para futuros desarrollos de la democracia: desde la generalización de los sistemas democráticos de gobierno a todos los estados del planeta, hasta la aparición de nuevas formas de organización democrática en diferentes niveles y sectores de la organización social: democracia en el gobierno mundial, democracia en la fábrica, en la escuela, en los partidos políticos o en las asociaciones de vecinos. Los otros dos ejes en los que se pueden producir desarrollos significativos de la democracia son el eje de la legitimación y el eje de la eficiencia social de los sistemas democráticos. Quizá podríamos 
asumir que hay una cuarta dimensión de la democracia, representado por el eje de la justicia o la equidad, pero me parece que esta dimensión, aunque esencial (la medida en que el procedimiento democrático contribuye o aumentar o a dificultar la justicia social: ver Vargas Machuca, 2002), se puede considerar como una función de la legitimación y la eficiencia social. Pues bien, mi propósito en estas páginas es argumentar en torno a la conveniencia de desarrollar la democracia en un ámbito específico que llamo la democracia tecnológica. Se trata de una propuesta de extensión de la democracia a un sector de la actividad social, lo que considero necesario, no sólo en virtud de sus propios merecimientos, por la importancia que la tecnología tiene para las sociedades actuales, sino también como una forma de responder a algunos problemas de legitimación y eficiencia social a los que se enfrentan los sistemas democráticos.

Todo el mundo está de acuerdo en atribuir una gran importancia a la tecnología como factor decisivo en la dinámica interna de las sociedades actuales. Eso explica la existencia también de un amplio consenso respecto a la importancia creciente de la políticas tecnológicas en la agenda de los gobiernos y de las organizaciones multinacionales. Pero sobre esta base común, se entrecruzan múltiples discursos y opciones políticas completamente diferentes. Para ordenar el panorama podríamos distinguir tres tipos de relaciones de la tecnología con la política: la tecnología como marco condicionante, como instrumento y como objetivo de la acción política.

\section{La sociedad tecnológica}

El discurso sobre la tecnología como marco condicionante de la acción política está muy extendido en nuestros días. Una buena parte de la literatura (y las políticas gubernamentales, muy asociadas a esa literatura) sobre la sociedad de la información o del conocimiento parte del supuesto de que las tecnologías actuales, en especial las nuevas tecnologías de la información y las comunicaciones (TIC) constituyen la base de un nuevo paradigma socioeconómico a partir de la cual se configura un sistema social con todo lo que ello implica en la esfera cultural, económica, y política. Las versiones más ingenuas de este discurso suelen asumir de forma bastante acrítica una posición veladamente determinista, cuya coherencia exigiría asumir que la tecnología evoluciona por su cuenta, fuera de control humano y que impone a la humanidad sus dictámenes respacte q cómo organizar la convi- 


\section{La democracia tecnológica}

vencia, la economía, la política. En algunas ocasiones la reflexión sobre las nuevas tecnologías se extiende hasta sus repercusiones sobre la organización de los sistemas democráticos. A este género, que en ocasiones parece próximo al de la ciencia ficción, pertenecen las especulaciones usuales sobre la democracia electrónica, el aumento de las posibilidades de participación en los asuntos públicos gracias a las TIC, el uso de Internet para mejorar la conexión entre los representantes políticos y sus ciudadanos, etc. Creo que algunas de estas especulaciones son importantes y contienen contribuciones valiosas para entender algunas características de las sociedades actuales. La noción de sociedad red que propone Castells (1997), por ejemplo, creo que permite captar de forma muy nítida algunas características esenciales del mundo actual. En mi opinión, sin embargo, muchas otras ideas sobre estos temas, a pesar del predicamento que a veces consiguen, son más ingenuas y llamativas que rigurosas y relevantes. Por decirlo de otra manera: no creo que los retos ni las soluciones más importantes para el desarrollo de la democracia tengan mucho que ver con el uso de tecnologías de la información para organizar votaciones, hacer continuas consultas a la población sobre cuestiones políticas o mejorar sustancialmente los problemas de legitimación y eficiencia que tienen los representantes políticos en su comunicación con los electores.

Una anécdota puede servir para transmitir y justificar mi escepticismo ante todas estas ilusiones de democracia informatizada. Hace unos años, el Senado español organizó una comisión de estudio sobre las tecnologías de la información y, por primera vez en el parlamentarismo español, se diseñó una página web en la que cualquier ciudadano podía acceder a toda la información recogida por la comisión, con los textos íntegros de las comparecencias de los expertos y de las intervenciones de los senadores. Además la web disponía de varios espacios (foros) para que los ciudadanos pudieran participar en el debate. La idea me pareció interesante $\mathrm{y}$, después de haber comparecido como experto en la comisión, tuve una gran curiosidad en saber si había mensajes del público en torno a los temas que allí habíamos tratado. Entré en el foro de cuestiones generales y me llevé una buena sorpresa: las primeras intervenciones eran críticas bastante desabridas a los políticos por meter las narices en un asunto como Internet, hasta entonces patrimonio de internautas, al parecer más apocalípticos que integrados. Bueno, desde luego no es más que una anécdota, que no hace justicia a la meritoria y pionera labor de esa comisión del Senado, pero creo que puede ser significativa. Lo que representa es la enorme distancia que existe entre los discursos teóricos sobre la sociedad de 
la información y sus repercusiones para la política democrática, y la efectiva realidad de ésta en las sociedades actuales.

Para resumir, no creo que la influencia de las TIC en la extensión o en la mejora de la democracia tenga que ser mayor o diferente que la del transporte por ferrocarril en los sistemas parlamentarios del siglo XIX. Desde luego, me imagino que a los representantes de provincias les facilitó mucho la vida el hecho de poder viajar desde sus circunscripciones a Madrid. Pero dudo de que esto alterara profundamente la cantidad y la calidad de la democracia, al menos en comparación con otros fenómenos sociales no tan directamente relacionados con las innovaciones tecnológicas de la época. Y supongo que algo parecido ocurrirá con las tecnologías actuales: en sí mismas son un buen instrumento de comunicación, $\mathrm{y}$ en esa medida pueden ser sumamente útiles a los electores y a los representantes políticos, lo mismo que lo son para los comerciantes y los taxistas. Pero cuando hablamos de democracia tecnológica no debe entenderse esto como democracia con móvil o con Internet.

Más interesante a este respecto son las otras dos relaciones que hemos señalado entre la tecnología y la política: la tecnología como instrumento y como objetivo de la acción política. Empecemos por la primera.

\section{Democracia tecnológica mínima}

Una primera forma de ver esta relación proviene de la consideración de la tecnología como protagonista del reino de los medios, frente a la moral y la ética política que se sitúan predominantemente en el reino de los fines. Por una parte los medios tecnológicos permiten alcanzar e incluso concebir fines para la acción política que de otra forma serían impensables. Las políticas sanitarias de vacunación obligatoria no fueron posibles sino después de que se inventaran las vacunas. En este aspecto ya se plantea un interesante problema desde el punto de vista del desarrollo de la democracia. Desde sus orígenes la democracia se ha considerado, moralmente, como el mejor sistema (para los liberales pesimistas, el menos malo) de gestión o gobierno de la sociedad. Este juicio moral se basa en una intuición muy valiosa: si asumimos que todos los individuos humanos tienen la misma dignidad y los mismos derechos básicos, cualquiera que sea la justificación que demos a la existencia del Estado o de cualquier otra forma de poder político, será siempre preferible un sistema de organización social en 
el que todos los individuos tengan iguales oportunidades de participar en el uso y control de ese poder para gestionar la sociedad. Pues bien, en la medida en que la tecnología aumenta las capacidades de actuación de una sociedad sobre su entorno, hay un sentido obvio de la extensión de la democracia, que consiste en garantizar el derecho de todos los ciudadanos a participar en las decisiones sobre el uso de las posibilidades tecnológicas en asuntos de interés público. La tecnología como instrumento para las políticas en un sistema democrático exige arbitrar los medios para que el público pueda entender las nuevas opciones que se le presentan gracias al desarrollo tecnológico, participar en su evaluación y contribuir a la formación de la opinión pública acerca de estos temas. Una variante de esta facultad es el derecho de todos los ciudadanos de acceder al conocimiento técnico y de contar con el juicio de los expertos como elemento fundamental para conformar la opinión pública y participar en las decisiones políticas sobre asuntos técnicamente complejos.

Este es, digámoslo así, el contenido mínimo de la democracia tecnológica. Su justificación es de sentido común. Una democracia en la que los ciudadanos tuvieran vetado el acceso al conocimiento tecnológico y al asesoramiento objetivo y honrado de los expertos, sería una democracia secuestrada por la oligarquía de los técnicos. Pero para ser coherentes, este contenido mínimo de los derechos de participación en la democracia tecnológica debería tener también una contrapartida en las obligaciones de los ciudadanos: no convertir en problemas políticos aquellos asuntos para los que existen soluciones técnicas solventes y contrastadas.

Hay algunas objeciones en contra de esta propuesta, que considero mínima, de democracia tecnológica. En primer lugar están las objeciones que se basan en la imposibilidad de que todos los ciudadanos accedan a la totalidad del conocimiento tecnológico relevante para cada una de las decisiones que se toman a través de procedimientos democráticos. Esta imposibilidad puede ser de dos tipos: pragmática o intrínseca. Por imposibilidad pragmática me refiero a aquella que se debe a razones de conveniencia política. Por ejemplo, puede que no resulte conveniente políticamente que todos los ciudadanos tengan acceso a cierta información técnica referida a la ubicación de depósitos de combustible nuclear susceptible de uso militar. Este tipo de imposibilidad o inconveniencia pragmática del acceso del público a todo el conocimiento tecnológico se puede argumentar con los mismos criterios con los que se argumenta a favor de la existencia de secretos de Estado o de información políticamente relevante pero no accesible a todos los ciu- 
dadanos. En las democracias representativas existen mecanismos contrastados para gestionar estos secretos de Estado y la limitación del acceso al conocimiento tecnológico basada en este tipo de argumentos no requiere justificaciones nuevas. Así que dejemos este asunto al margen de nuestra discusión. Más importante para nosotros es la cuestión de si existen limitaciones intrínsecas para el acceso de todos los ciudadanos al conocimiento tecnológico que se necesita para determinadas decisiones políticas en un sistema democrático. La base de este argumento es el carácter especializado y difícilmente comprensible del conocimiento tecnológico avanzado y el consiguiente riesgo de deformación de la información que se transmite al público con las consecuencias que eso puede tener a la hora de tomar decisiones basadas en una información incorrectamente procesada o comprendida. Se trata de una objeción seria e importante, que está en la base de muchas prácticas comunes en la política tecnológica de los países industrializados, en especial en las políticas relacionadas con tecnologías "sensibles» como la energía nuclear. Estas políticas se han apoyado en el secretismo y en la desconfianza respecto a la capacidad del público para comprender todas las implicaciones de los problemas que había que afrontar. El resultado ha sido desastroso: la desconfianza del público se ha generalizado y ha hecho prácticamente imposible seguir adelante con determinados proyectos tecnológicos, a veces con consecuencias desastrosas para la propia seguridad de los ciudadanos atemorizados, como ocurre, por ejemplo, con la gestión de residuos nucleares en todos los países que tienen plantas de producción de este tipo de energía. Sin embargo, en las tradiciones democráticas existen otros precedentes aplicables a este tipo de problemas. Para la mayoría de los ciudadanos (y para muchos de sus representantes políticos) los tecnicismos jurídicos o económicos de algunos debates parlamentarios son tan incomprensibles como las ecuaciones de Maxwell, pero eso no les impide participar y seguir esos debates, directamente o a través de sus representantes y con la ayuda de las organizaciones (partidos políticos) intermediarias. No debería ser difícil generalizar esta experiencia de intermediación a cualquier otro ámbito del conocimiento técnico especializado, más allá de los límites de la tecnología jurídica o de la cháchara económica.

Una última objeción se refiere a la dificultad que a veces existe de trazar el límite entre conocimiento técnico y opinión política. La existencia y extensión de las controversias públicas acerca de cuestiones aparentemente tecnológicas demuestra que tal delimitación no siempre es posible. En el caso extremo podría derivarse de aquí una deslegitimación del conocimiento tecnológico en general: en realidad todo vale 
y lo importante no es el conocimiento técnico sino el poder para imponer una opinión. No podemos entrar aquí en todos los interesantes problemas epistemológicos y sociales que plantea esta nueva moda irracionalista que ha proliferado en algunos ambientes académicos. Pero para nuestros propósitos basta con advertir que el hecho de que existan controversias tecnológicas no nos autoriza a identificarlas con controversias políticas. Las primeras se refieren a la forma más eficiente de obtener un resultado que se considera valioso; las segundas se centran en la elección del tipo de resultados que vamos a asumir como valiosos o en la cantidad de valor que vamos a atribuir a los resultados que se nos ofrecen como posibles. En muchas ocasiones durante el debate se mezclan los dos tipos de cuestiones, pero el criterio para saber si la cancelación del debate, cuando se produce, ha șido de carácter predominantemente técnico o político es sencillo: en el primer caso los problemas se resuelven "calculando», en el segundo «negociando». De cualquier forma, en la mayoría de las ocasiones el conocimiento tecnológico disponible, no sometido a controversia, sino aceptable por todo el mundo, es suficiente para resolver muchos problemas que deberían quedar así sustraídos al debate político. Esto sucede hoy en día en todas las esferas de la actuación política, aunque en muchas de ellas no interese reconocerlo. Por eso, el contenido mínimo de la democracia tecnológica que proponemos incluye también un principio de sabor tecnocrático: si existe una solución técnica adecuada para un problema, no lo complique usted transformándolo en un problema político.

Para que la democracia tecnológica, con este contenido mínimo, sea posible y funcione adecuadamente, es preciso introducir cambios importantes tanto en el entramado institucional de la democracia, como en la cultura cívica de los ciudadanos y en las prácticas y hábitos de la participación política. Pero esto es sólo - recordemos- el contenido mínimo de la democracia tecnológica.

\section{Democracia tecnológica plena}

Decíamos que hay un tercer tipo de relaciones de la tecnología y la política en las que aquella aparece no como entorno ni como instrumento, sino como objetivo de la acción política. Pues bien es en este ámbito donde hay que situar, en mi opinión, el contenido máximo o completo de la democracia tecnológica. Lo diré nuevamente con palabras del mismo ensayo de Lafontaine: 
«Dado que el progreso técnico no entra en razón por sí solo, somos nosotros quienes hemos de obligarle a que lo haga. Para restablecer un consenso racionalista del progreso en la sociedad, es preciso consensuar la Técnica» (p. 193)

Podríamos definir sucintamente el contenido máximo de la democracia tecnológica con estas mismas palabras: «es preciso consensuar la técnica». Lo que implica, de forma más explícita, reivindicar la democracia tecnológica plena, entendida como el derecho de todos los ciudadanos a participar en las decisiones acerca del desarrollo y control de la tecnología. Se tata de poder participar no solo en las decisiones acerca de qué debemos hacer a partir de las posibilidades que nos ofrece la tecnología disponible, sino también en las decisiones acerca de qué queremos poder hacer en el futuro gracias a las tecnologías que hoy nos proponemos desarrollar.

Hay básicamente dos formas de entender la intervención política en la dirección y el control del desarrollo tecnológico. La primera es una forma que llamaremos subsidiaria. Se supone que el desarrollo tecnológico se produce impulsado (o atraído) por fuerzas sociales, culturales y fundamentalmente económicas, que son las responsables del flujo continuo de innovaciones técnicas, es decir de nuevos productos y procesos que entran en el circuito de la producción y distribución de bienes y que tienen su origen en conocimientos científicos e invenciones tecnológicas. El poder político tiene la posibilidad de contribuir a alentar. apoyar e incentivar esos procesos de desarrollo tecnológico y a facilitar la obtención de ventajas económicas y sociales a partir de ellos. En la actualidad todos los gobiernos tienen líneas de actuación política en este terreno y es ampliamente compartida la doctrina que rige en la regulación política de tales procesos de desarrollo tecnológico. Más allá de pruritos ideológicos acerca de los límites de la política y del mercado, todo el mundo acepta la necesidad de que el poder público intervenga en este ámbito que se considera demasiado importante y arriesgado como para dejarlo en manos de la estricta economía de mercado. Pero no es aquí donde se plantea actualmente el debate sobre la política tecnológica. Como hemos visto en un capítulo anterior, junto a las políticas de promoción e incentivos a la innovación tecnológica que denominamos subsidiarias, se han desarrollado también políticas sustantivas de orientación, evaluación y control público del propio proceso de desarrollo tecnológico. También en esto hay un amplio consenso: los estados no sólo promocionan la ciencia y la tecnología, sino que procuran establecer prioridades y orientar el desarrollo tecnológico en determinadas direcciones en vez de en otras. La alternativa aquí no 


\section{La democracia tecnológica}

está entre intervención estatal y liberalismo puro, sino entre criterios de intervención democráticos y no democráticos. Veamos cuál es la diferencia.

En la actualidad, una cantidad creciente de las decisiones que condicionan el desarrollo de la tecnología se toman en los consejos de administración de las grandes empresas multinacionales en función de una combinación de criterios en la que el peso fundamental lo aportan los criterios de rentabilidad económica de carácter más o menos coyuntural o estratégico y el secundario, aunque relevante, los criterios de eficiencia y novedad tecnológica. Esta combinación de criterios suele conducir de hecho a un tipo de desarrollos tecnológicos que no tienen por qué coincidir con los intereses y modelos de desarrollo que los ciudadanos preferirían si tuvieran la oportunidad de intervenir en su definición. El problema es que éstos, los ciudadanos, a duras penas podrán percibir su verdadera situación: en relación con la tecnología, pierden su condición de ciudadanos y se ven reducidos a simples consumidores o usuarios. $\mathrm{Y}$ además se ven compelidos a pensar que eso es así por la propia naturaleza de las cosas, y no por la falta de cauces para la participación en las decisiones estratégicas que determinan las formas y direcciones del desarrollo tecnológico. Pues bien, en este escenario la intervención política, si no tiene un fuerte componente de participación democrática, se limitará a priorizar aquellas áreas o líneas de desarrollo que son más rentables desde el punto de vista económico o más necesarias desde el punto de vista instrumental (por ejemplo, en función de las políticas de defensa). En este punto surge la pregunta de si es realmente razonable pretender que la dirección del desarrollo tecnológico se decida democráticamente, más allá del valor democrático que pueda tener la lógica propia del mercado.

Podríamos extendernos largamente en la discusión de este tipo de cuestiones. Pero creo que bastará con un único argumento que no es muy usual en este contexto, aunque a mí me parece definitivo. Lo mismo que en general se suele argumentar a favor de la intervención pública en aquellos aspectos de la actividad económica en los que el mercado por si solo conduciría a ineficiencias insuperables, creo que también se puede argumentar a favor de la participación democrática en el control y orientación de aquellos procesos que, si se dejan a su propia lógica interna o se controlan políticamente al margen de los procedimientos democráticos, conducen necesariamente a una reducción significativa de la capacidad de los ciudadanos para hacerse responsables de su propio destino, lo que implica una pérdida irreparable de libertad y autonomía. 
Pues bien, creo que hay una lógica interna en el desarrollo tecnológico que lleva a esa pérdida irreparable de libertad y autonomía, aunque bien es cierto que compensada con una mayor facilidad de acceso a los bienes y servicios generados gracias a la tecnología. La razón de este fenómeno es sencilla: la facilidad de acceso al uso y consumo de bienes tecnológicos es tanto mayor cuanto más transparente es al usuario la propia tecnología que utiliza. Con otras palabras: el éxito tecnológico es una función directa de la alienación del consumidor. Para que una tecnología penetre en el mercado debe ser fácil de usar, de reparar, de mantener y hasta de desechar. Todo ello se consigue a costa de la facilidad para penetrar en su interior. Los microchips son máquinas maravillosas en sí mismas que desempeñan multitud de funciones inteligentes y pueden integrarse en casi cualquier dispositivo técnico mejorando su rendimiento. A cambio su estructura interna permanece opaca al resto del sistema, al usuario e incluso al diseñador. Se puede sustituir un microchip, pero no se puede reparar; mucha gente lo puede utilizar pero nadie por sí solo lo puede construir; es posible que todo el mundo entienda lo que hace pero que nadie sepa realmente de forma completa cómo lo hace (cada uno de los ingenieros que ha participado en su diseño sólo conoce una parte del mismo y quienes han ensamblado el conjunto sólo necesitan conocer los flujos de entrada y salida de cada subsistema, no su estructura interna).

Naturalmente la participación democrática en el diseño y el control del desarrollo tecnoiógico no debería hacerse a costa de la eficiencia y la capacidad difusiva de las tecnologías. Pero en muchas ocasiones una presencia más directa del consumidor en las fases de diseño, orientación y control de la tecnología, ejerciendo las funciones de ciudadano responsable y dueño de su destino, podría alterar profundamente su relación con la tecnología. Y también podría suceder que los esfuerzos de las empresas por responder a este nuevo "vigilante tecnológico» dieran lugar a innovaciones tecnológicas no sólo más eficientes y novedosas sino incluso también más rentables. En la actualidad el catalizador de gases de la combustión es obligatorio en todos los automóviles, y ha contribuido muy positivamente a asimilar la cultura del automóvil en la cultura de la preocupación por el medio ambiente. En su origen fue una reivindicación ecologista que se abrió paso hacia una de las ramas industriales más poderosas del siglo XX a través de las instituciones democráticas. ¿Por qué no desear que suceda lo mismo en la regulación de la biotecnología, la terapia génica, la investigación médica en células madre, etc.? Creo que es legítimo, en este contexto, preguntarnos qué habría sucedido si el rápido y abigarrado 
despliegue de antenas de telefonía celular que se ha producido en España hubiera sido consensuado a través de cauces democráticos más participativos. Por el momento sabemos las consecuencias de no haberlo hecho así: los ciudadanos desconfían irracionalmente de las antenas, las empresas han cometido errores no sólo de «relaciones públicas» sino también de diseño técnico ( $\mathrm{y}$ estético), y el coste final de toda la operación se ha encarecido con miles de millones dedicados a revisar la emisión de radiación para tranquilizar al público, aunque todos los expertos saben de antemano que las antenas instaladas cumplen las especificaciones técnicas y legales y que, dentro de esos límites, son inocuas.

La democracia tecnológica plena consiste en el derecho de todos los ciudadanos a acceder a todo el conocimiento tecnológico relevante para la toma de decisiones en asuntos de interés público y a participar en el diseño, evaluación y control del desarrollo tecnológico. En las sociedades actuales esta última frontera en el largo camino de la extensión de la democracia no es una posibilidad abierta, es una necesidad ineludible.

\section{Qué se puede hacer}

La extensión de la democracia requiere cambios tanto institucionales como culturales. En el plano institucional la democracia representativa todavía tiene un amplio margen de adaptación y maniobra para dar cabida a las nuevas necesidades y sobre todo para ensayar nuevas experiencias. En el plano cultural los medios de comunicación jugarán sin duda un papel decisivo en la extensión de la cultura tecnológica a todos los ciudadanos. Pero lo que ahora pretendo es apuntar algunas ideas acerca de los contenidos de cultura tecnológica que podrían ayudarnos en la tarea de extender la democracia al control y el dominio de la tecnología.

El nacimiento y desarrollo de los sistemas democráticos liberales fue acompañado de grandes cambios culturales. Para que los que habían sido siervos pudieran sentirse ciudadanos tuvieron que poder entender que su condición de siervo no se debía a la naturaleza de las cosas, sino a las relaciones de dominación impuestas por individuos cuya fuerza real tenía, en última instancia, el mismo origen (la voluntad humana) que la capacidad de resistencia frente a su imposición. La historia de la democracia, como la del movimiento obrero, la de las sufragistas o la de los movimientos de liberación anticoloniales en el 
siglo XX, son historias de cambios culturales: son la historia de la generalización de la enseñanza, de la capacitación técnica de los trabajadores, del acceso de la mujer a los estudios, de la formación de la elites dirigentes de las colonias en las universidades de las metrópolis. La extensión de la democracia siempre ha ido acompañada por una extensión y transformación de la cultura. La democracia tecnológica también requiere profundos cambios culturales. La cuestión es saber si podemos vislumbrar de antemano la dirección en que deben producirse esos cambios. He aquí algunas ideas tentativas sobre posibles contenidos de la cultura tecnológica que necesitaríamos construir.

\section{El uso y la apropiación de las tecnologías}

Las ideas predominantes en las sociedades actuales acerca de la propiedad y el uso de la tecnología siguen ancladas, en lo fundamental, en las mismas ideas con las que el derecho romano resolvía los conflictos entre propietarios agrícolas: la propiedad de un bien material garantiza el derecho de uso y abuso del mismo sin más limitaciones que las derivadas de las propiedades de los vecinos. El derecho de patentes y de propiedad intelectual ha introducido una buena dosis de «desmaterialización» en ese concepto tradicional, pero básicamente responde a los mismos principios. En cambio, el uso y la propiedad de la tecnología actual no encajan bien en ese patrón. El fenómeno «Napster» y sus secuelas es revelador de la nueva situación: la copia para uso privado de una producción intelectual o artística, sin fin alguno de lucro, se puede transformar en una inmensa red de distribución que priva de toco valor económico al derecho de propiedad intelectual. La respuesta espontánea ante esta situación es intentar "poner puertas al campo", lo cual resulta del todo inútil: por cada sistema técnico de protección contra copias que aparece, surge un antídoto para desactivarlo. Las únicas opciones realmente viables implican respuestas institucionales y jurídicas para las que se necesita abrir un proceso de negociación y de debate político.

Es evidente que la investigación para el desarrollo de vacunas y medicamentos contra el sida requiere que las compañías farmacéuticas puedan tener expectativas razonables de obtener beneficios económicos. Pero no es posible mantener todas las restricciones a la difusión de una tecnología que permite el derecho de patente, si eso hace que el sistema en su conjunto pierda rápidamente su legitimidad, como puede suceder si la comunidad internacional condena a la muerte a millones 


\section{La democracia tecnológica}

de habitantes de países pobres por no autorizar la fabricación y distribución a bajo precio de los medicamentos adecuados.

No es fácil vislumbrar nuevos principios capaces de regular el uso y la apropiación de las tecnologías que hagan compatible el acceso de los ciudadanos a las nuevas posibilidades tecnológicas y la existencia de incentivos para la investigación y la innovación. Pero en la propia cultura tecnológica de nuestro tiempo existen experiencias que apuntan en una dirección prometedora: el movimiento del software libre, la regulación de sistemas de protección jurídica frente a la apropiación privada de productos tecnológicos que son resultado de un trabajo colectivo, la existencia de obras de creación colectiva sin derechos de autor reconocidos, etc. Lo que se vislumbra en el horizonte es una nueva forma de percibir la propiedad de la tecnología, como una condición que te permita usarla y disfrutar de ella, e incluso, en su caso, reclamar un reconocimiento social por haber contribuido a su desarrollo, pero sin que ello implique que puedas impedir que otros la usen y disfruten o la modifiquen y mejoren. Pekka Himanen (2001) ha acuñado una feliz expresión para recoger muchos de estos elementos de la nueva cultura tecnológica: La ética del Hacker. Todavía no sabemos cómo será un mundo así, pero no cabe duda de que en él la democracia tecnológica plena será más viable.

\section{Tecnologías entrañables}

Ya hemos aludido a la paradoja que supone el hecho de que, en general, cuanto más fácil de usar es una tecnología, más incomprensible resulta para el usuario: las tecnologías transparentes exigen ciudadanos ciegos. Hay que reconocer en este punto una notable peculiaridad de la cultura tecnológica predominante en la actualidad. En otras épocas podía suceder que un inventor o un gobierno quisiera mantener en secreto su tecnología para impedir que se difundiera y evitar así perder la ventaja que su posesión le daba, en el plano militar, económico, político, etc. En la actualidad, sin embargo, las tecnologías se hacen opacas al usuario para facilitar su uso y su difusión. Este es, en efecto, el significado de conceptos como el de «interfaz transparente al usuario» o «tecnología amigable». El usuario, el consumidor, debe ser capaz de usar y disfrutar una tecnología sin necesidad de entenderla; una innovación con vocación de difusión universal debe aspirar a ser compatible con todas las culturas y, para ello, lo mejor es que no requiera que el usuario incorpore nuevos conocimientos, que las prácticas de 
uso sean extremadamente simples y que los valores incorporados sean fácilmente asimilables. En definitiva, que la tecnología no resulte extraña. La forma más rápida y fácil de conseguir esto es «dorar la píldora»: esconder el contenido de la tecnología y mostrarle al usuario solamente una superficie dorada, una interfaz amigable, una tecnología tan fácil de usar como imposible de desentrañar.

Durante años se ha considerado que precisamente esta tendencia hacia las tecnologías amigables constituía un gran avance en la humanización de la tecnología. Pero hay razones para pensar que se trataba de un error. Seguramente inspirado por la doble convicción profunda de que en sí mismas las tecnologías son inhumanas (y por eso requieren ser "humanizadas» en la interfaz de usuario) y de que por principio los consumidores son estúpidos (y por eso hay que tratarles como tales, negándoles el acceso a las entrañas de las tecnologías que utilizan).

Pero los errores se pagan. Los sistemas amigables de interfaz entre el usuario y los ordenadores han terminado cargando sobre estas máquinas la responsabilidad de todos los errores humanos que se cometen con ellas. El resultado es la deslegitimación social de un sistema que se considera ajeno a los intereses humanos y dominado por la lógica interna de una tecnología inasequible. Si renunciamos a hacernos responsables de nuestras máquinas, no podemos después quejarnos de su ineficiencia. A fuerza de hacer máquinas inteligentes y opacas, terminaremos haciendo realidad la sospecha de que el usuario es estúpido.

No sé cómo se resolverán estas paradojas de nuestra cultura tecnológica. Pero me gustaría proponer la sugerencia de construir tecnologías que no sólo sean fáciles de usar sino sobre todo atractivas para integrar en la vida personal o social. Esto es lo que quiero sugerir con la idea de tecnologías entrañables: asimilables, amables, integrables en la propia vida, que se pueda disfrutar de ellas no solo usándolas a ciegas, sino apropiándose de su lógica interna, comprendiéndolas.

¿Por qué se considera entrañable un paisaje de molinos de viento o la silueta de un viejo molinete metálico al lado de un pozo, pero no un parque eólico de aerogeneradores de energía eléctrica? ¿Por qué el teléfono móvil se ha convertido rápidamente en un complemento personal, como la corbata o el bolso, pero seguimos viendo la infraestructura física de la telefonía celular como algo completamente ajeno a nuestros intereses? ${ }^{1}$

La democracia tecnológica requiere no sólo que la gente normal pueda tener acceso a conocimientos e informes técnicos comprensibles, sino que la propia tecnología pueda integrarse plenamente (con todo su contenido) como un elemento normal de la cultura de la gente. 


\section{La democracia tecnológica}

\section{Notas}

1 Una sugerencia para la inflexión desde el constructivismo social: el éxito social de la bicicleta podría estar relacionado con el hecho de que se trata del más entrañable de los dispositivos mecánicos de transporte. En la actualidad, si quieres disponer de un buen automóvil tienes que renunciar a jugar a la mecánica con él. Con una motocicleta aumentan un poco las posibilidades de contribuir activa y creativamente a su cuidado. Pero las bicicletas son otra cosa: hasta hace unos años, al menos, todo en ellas era diáfano y claro, no escondían secretos tecnológicos y más bien requerían la activa participación continua del usuario para mantener sus prestaciones. En Broncano (2000) hay interesantes reflexiones y originales ideas para una teoría de la humanidad de la técnica, es decir de las tecnologías entrañables.

\section{Referencias bibliográficas}

Broncano, Fernando (2000). Mundos artificiales, Paidos, México, Barcelona Himanen, Pekka (2002) La ética del hacker, Destino, Barcelona

LAFONTAINE, Oskar (1989): La sociedad del futuro, Sistema, Madrid.

VARGAS-MACHUCA, Ramón (2002): El liberalismo republicano, los modelos de democracia y la causa del reformismo. En: Rubio Carracedo, J., Rosales, J.M, Toscano Méndez, M. (comps.): Retos pendientes en ética y política, Trotta, Madrid, 2002.

Castells, Manuel (1997): La era de la información. Economía, sociedad y cultura.

Vol, 1: La sociedad red. Alianza Editorial, Madrid. 